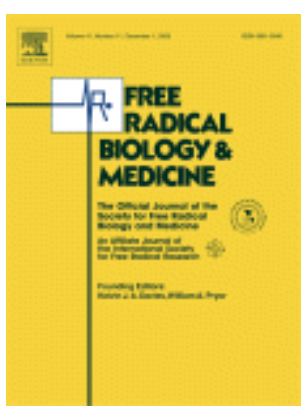

Original Contribution

\title{
Protection against oxidative stress through SUA7/TFIIB regulation in Saccharomyces cerevisiae
}

- Joana Paes de Faria ${ }^{a}$,

- $\quad$ Lisete Fernandes ${ }^{a}$, $\underline{b}$,

- anstituto Gulbenkian de Ciência, Rua da Quinta Grande, 6 Apartado 14, 2780156 Oeiras, Portugal

- ${ }^{b}$ Escola Superior de Tecnologia da Saúde de Lisboa, Lisboa, Portugal

\section{Abstract}

The general transcription factor TFIIB, encoded by SUA7 in Saccharomyces cerevisiae, is required for transcription activation but apparently of a specific subset of genes, for example, linked with mitochondrial activity and hence with oxidative environments. Therefore, studying SUAT/TFIIB as a potential target of oxidative stress is fundamental. We found that controlled SUA7 expression under oxidative conditions occurs at transcriptional and mRNA stability levels. Both regulatory events are associated with the transcription activator Yap1 in distinct ways: Yap1 affects SUA7 transcription up regulation in exponentially growing cells facing oxidative signals; the absence of this activator per se contributes to increase SUA7 mRNA stability. However, unlike SUA7 mRNA, TFIIB abundance is not altered on oxidative signals. The biological impact of this preferential regulation of SUA7 mRNA pool is revealed by the partial suppression of cellular oxidative sensitivity by SUA7 overexpression, and supported by the insights on the existence of a novel RNA-binding factor, acting as an oxidative sensor, which regulates mRNA stability. Taken together the results point out a primarily cellular commitment to guarantee SUA7 mRNA levels under oxidative environments. 


\section{Abbreviations}

- 5-FOA, 5-fluoroorotic acid;

- GTFs, general transcription factors;

- MOPS, 4-morpholinepropanesulfonic acid;

- $\mathrm{PCR}$, polymerase chain reaction;

- PMSF, phenylmethylsulfonyl fluoride;

- SC, synthetic complete;

- SDS-PAGE, sodium dodecyl sulfate-polyacrylamide gel electrophoresis

\section{Keywords}

- TFIIB;

- Oxidative stress signaling;

- Yap1;

- mRNA stability

Corresponding author. Instituto Gulbenkian de Ciência, Rua da Quinta Grande, 6 Apartado 14, 2780-156 Oeiras, Portugal. Fax: +351214407970.

Copyright @ 2006 Elsevier Inc. All rights reserved. 